\title{
Review Article \\ Review of Low Profile Substrate Integrated Waveguide Cavity Backed Antennas
}

\author{
Guo Qing Luo, Tian Yang Wang, and Xiao Hong Zhang \\ Microelectronic CAD Center, Hangzhou Dianzi University, Hangzhou 310018, China \\ Correspondence should be addressed to Guo Qing Luo; gqluo@emfield.org
}

Received 12 September 2013; Accepted 12 October 2013

Academic Editor: Bing Liu

Copyright ( 2013 Guo Qing Luo et al. This is an open access article distributed under the Creative Commons Attribution License, which permits unrestricted use, distribution, and reproduction in any medium, provided the original work is properly cited.

\begin{abstract}
Low profile cavity backed antennas (CBA) based on substrate integrated waveguide (SIW) technology presented in published papers have been reviewed in this paper. Their operating mechanisms have been discussed and methods for improving the performance, such as bandwidth enhancement, size reduction, and gain improvement, have been presented. These novel antennas retain the advantage of conventional metallic cavity backed antenna, including high gain, high front-to-back ratio, and low cross polarization level, and also keep the advantages of planar antenna including low profile, light weight, low fabrication cost, and easy integration with planar circuit.
\end{abstract}

\section{Introduction}

Conventional metallic cavity backed antennas (CBA) have been extensively presented for their satisfactory radiation performances $[1,2]$. Figure 1 shows the classical conventional CBA, and the depths of their metallic backed cavities are roughly one-quarter wavelength. Their bulky volumes make the conventional metallic CBA not suitable for some practical applications.

Substrate integrated waveguide (SIW) technique has been developed to design microwave and millimeter wave components and subsystems. SIW structure keeps the advantages of conventional metallic waveguides, such as high $Q$-factor, high selectivity, cutoff frequency characteristic, and high power capacity. It also has the advantages of low profile, light weight, conformability to planar or curved surfaces, and easy integration with planar circuits. In order to achieve low profile CBA, SIW is firstly adopted into CBA designs [3-5]. The backed cavity can be realized by using metallized via arrays through a single or multilayer substrate (referred to as SIW). In order to make the SIW cavity equivalent to the conventional metallic cavity, the conditions of $d / d_{p} \geq 0.5$ and $d / \lambda_{0} \leq$ 0.1 must be satisfied, where $d, d_{p}$, and $\lambda_{0}$ are the metallized vias diameter, the spacing between two neighboring vias, and the free space wavelength, respectively.
SIW cavity backed antennas are generally comprised of two types: SIW cavity backed patch antenna (shown in Figure 2(a)) and SIW cavity backed slot antenna (shown in Figure 2(b)). Both of these low profile SIW cavity backed antennas cannot only be fed by planar transmission lines such as microstrip line, coplanar waveguide (CPW), and grounded coplanar waveguide (GCPW) but also be fed by non-planar transmission lines such as probe and waveguide. In this paper, SIW cavity backed antennas, with different configurations, different feeding structures, and different performances, presented by international researchers are reviewed and their performance improvement methods are also discussed.

\section{SIW Cavity Backed Antenna}

SIW used in antenna design was firstly introduced in [6], in which SIW slot array antennas were designed similar to classical slotted waveguides antennas by etching longitudinal slots on the top metal surface of their substrates. SIW cavity backed antennas were also firstly presented in [3] with the form of cavity backed slot antenna.

SIW cavity backed slot antenna with linearly polarized performance was proposed in [4], in which a $50 \Omega$ GCPW line is adopted as a feeding element to stimulate a square SIW 


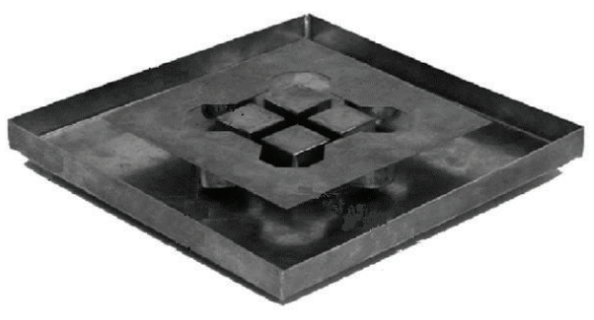

(a)

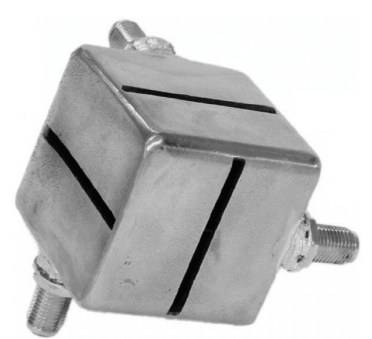

(b)

FIgURE 1: Conventional metallic cavity backed antennas.

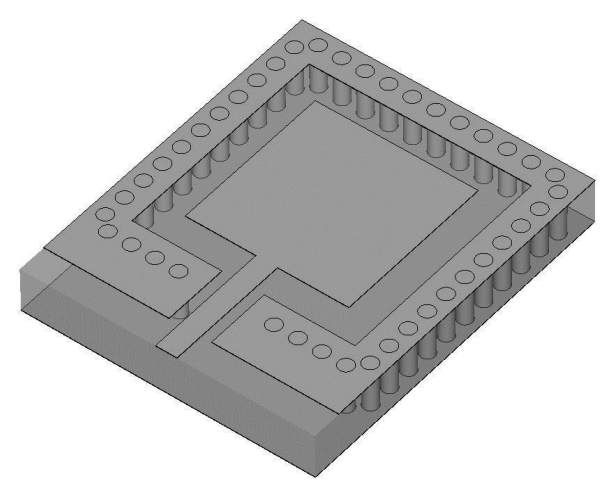

(a)

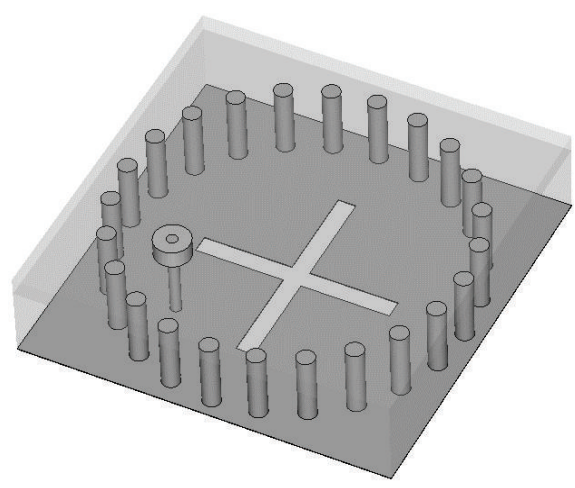

(b)

FIGURE 2: Low profile SIW cavity backed antennas: (a) patch antenna; (b) slot antenna.

backed cavity and a resonating slot etched at the center of the SIW backed cavity metal surface is used as the radiating element. When the square SIW cavity was in $\mathrm{TE}_{120}$ resonating mode, the dominant electric field at the two sides of the slot has opposite phase. Then, there is transverse electric field across the slot and thus energy can radiate into space by the slot. Another linearly polarized SIW cavity backed slot antenna was proposed in [7]. Its radiation was generated from a rectangular one point shorted slot when the antenna was fed by a coax-to-SIW transition. The concept of SIW cavity backed slot antenna applied in on-chip antenna design was investigated in [8], in which a fully on-chip antenna operating at $140 \mathrm{GHz}$ was fabricated with a standard CMOS technology. Its radiating slot was backed with an extremely thin cavity formed by two CMOS inner metal layers and the through metal via arrays. The SIW backed cavity prevents radiation from going into the lossy silicon substrate. Its radiation efficiency of $18 \%$ and gain of $-2 \mathrm{~dB}$ were achieved, which has an evident enhancement compared with those of conventional silicon on chip antennas. A dual frequency dual linearly polarized SIW cavity backed slot antenna was studied in [9]. A crossed slot, whose arms are parallel to the rectangular SIW cavity edges, is etched at the center of one SIW backed cavity metal surfaces. A single GCPW located at one diagonal line of the SIW cavity is employed as the feeding element to stimulate the cavity. When two cavity modes $\mathrm{TE}_{120}$ and $\mathrm{TE}_{210}$ were successfully excited, radiation from each arm was generated at a different frequency and each one has a different linear polarization. As one of the most important parameters of a dual frequency antenna, the frequency ratio $r_{f}$ of the proposed antenna is less than 2 .

SIW cavity backed slot antennas with circularly polarized radiation were firstly discussed in $[3,5,9]$, whose SIW backed cavity with two dimensions rotational symmetries was requisite. Crossed slot at the center of one metal surface of the SIW backed cavity was adopted as a radiating element. A single GCPW or probe located at one diagonal line of the crossed slot was used to excite the cavity. In order to simultaneously excite the two degenerate modes in the cavity by a single feed, a small perturbation of lengths of the crossed slot two arms must be introduced. When two orthogonal degenerate cavity modes $\left(\mathrm{TM}_{110}\right.$ or $\mathrm{TE}_{120}$ and $\left.\mathrm{TE}_{210}\right)$ were successfully excited, circularly polarized radiation was produced by tuning the two arms' length difference to achieve a $90^{\circ}$ phase difference between the radiations from the two arms. A SIW fed SIW cavity backed ring slot antenna for circularly polarized application was presented in [10]. Its circularly polarized radiation was generated by shorting the top patch and bottom ground plane using via. A broadband impedance matching was accomplished by inserting inductive via arrays at the input port. An X-band circularly polarized SIW cavity backed square ring slot antenna was proposed in [11], whose circularly polarized wave was generated by a combination of a shortened square ring slot etched in the SIW top wall and a shorting via placed between the area bounded by the ring and the SIW bottom wall. 
Another type of SIW cavity backed antenna was developed in [12]. The proposed SIW cavity backed patch antenna was comprised of a stack of two substrates, a microstrip substrate, and a cavity substrate. A square patch was printed on the microstrip substrate and fed by a microstrip line. The ground of the microstrip substrate and the top layer of the cavity substrate had a circular opening underneath the patch. Circular SIW backed cavity was constituted by metallized via array spaced along the circular opening. The proposed antenna exhibits a high-efficiency performance with an aperture radiation efficiency of better than $70 \%$ over a wide frequency range. A simple SIW cavity backed patch antenna presented by the same authors is shown in [13], which was fully constructed on a single substrate. A probe fed square patch was printed on the top conductor layer of the microstrip substrate and the top conductor layer also had a square opening around the patch. Metallized via arrays spaced along the square opening constituted the SIW cavity to back the radiating patch. The proposed antenna had a higher aperture radiation efficiency of $80 \%$ and a higher gain had been achieved.

SIW cavity backed patch antenna with circularly polarized radiation was proposed in [14]. Its circular polarization was generated by a conventional perturbed circular patch with a shorted-end via. The SIW cavity is used as a background to get a higher gain and a lower back lobe radiation. The proposed antenna was fed by SIW with an impedance matching network integrated with an asymmetric inductive diaphragm using via arrays and a broadband-matched impedance bandwidth of $17.32 \%$ had been achieved. A broad circularly polarized SIW fed patch antenna with $3 \mathrm{~dB}$ axial bandwidth of $7.1 \%$ was investigated in [15]. The proposed antenna comprised of two layers, the top patch layer and the bottom SIW layer. The metal sheet between these two layers is a ground plane for the patch. The patch is fed from the waveguide through two apertures at the ground plane by two probes. Circular polarization was produced with a proper choice of the locations of the two probes.

\section{Bandwidth Enhancement of SIW CBA}

Low profile SIW cavity backed antennas have a drawback of narrow bandwidth for their compact sizes. Bandwidthenhanced method should be studied in order to make them suitable for practical applications. A bandwidth-enhanced linearly polarized SIW cavity backed slot antenna was presented in [16], which was a single GCPW fed rectangular SIW cavity backed long slot antenna. The long slot was a completely matching slot other than the previously used resonating slot and its length is far more than a half resonant wavelength. After properly setting, two hybrid modes can be excited in the SIW cavity, which were two different combinations of the $\mathrm{TE}_{110}$ and $\mathrm{TE}_{120}$ resonances. One is a combination of a strong $\mathrm{TE}_{120}$ resonance and a weak $\mathrm{TE}_{110}$ resonance and the other is a combination of a strong $\mathrm{TE}_{110}$ resonance and a weak $\mathrm{TE}_{120}$ resonance. Although the fields distributing at the two sides of the slot are in phase, their huge magnitude difference also can make the slot radiate electromagnetic wave. Radiations generated from the two hybrid modes are consistent because their equivalent currents distributed in the slot are nearly the same. Compared with those of the previously presented SIW cavity backed slot antenna, fractional impedance bandwidth of the proposed antenna was improved about 4 times. Its gain and radiation efficiency are also slightly improved about $0.6 \mathrm{~dB}$ and $8 \%$, respectively. Its SIW cavity size is reduced about $30 \%$. A probe fed SIW cavity backed slot antenna using a via hole above the slot was studied in [17]. Two parallel cavity edges of the rectangular SIW backed cavity had a same quarter-wavelength length in the direction of perpendicular to the slot. A dog-bone shape half wavelength slot was used for an inductive loading of the slot to decrease the slot length. A via hole was placed above the slot for the dual resonance and then a $60 \%$ bandwidth had been achieved. Instead of using a via hole above the slot, the removal of the substrate underneath the slot also can be used to broaden the matched impedance bandwidth of an SIW cavity backed slot antenna [18]. When the substrate underneath the dog-bone shape slot was removed, the quality factor of the antenna resonator decreased and then a bandwidth enhancement of $24 \%$ and a radiation efficiency improvement of $6.2 \%$ had been achieved. A wideband SIW backed wide slot antenna was proposed in [19], which consisted of an aperture for radiating on the top surface of the top substrate and two rectangular SIW cavities with different sizes for mode generating and coupling that is embedded in the multilayer substrate. It exhibited a measured matching impedance bandwidth of $12.1 \%$.

A broadband SIW cavity backed patch antenna was developed in [20], which was a coplanar stripline (CPS) fed SIW cavity backed folded dipole antenna. By observing the magnetic field distribution figures, it can found that an additional half open cavity resonance was introduced besides the folded dipole inherent resonance. The proposed antenna had a broadband fractional matching impedance bandwidth of more than $30 \%$ by tuning the two different resonances. Radiations generated by these two resonances were also consistent. A bandwidth enhanced SIW cavity backed patch array was presented in [21]. The proposed array consisted of two stacked substrates. Patch elements were printed on the top microstrip substrate and the SIW backed cavities were constructed by many via holes spaced along circular openings at the bottom cavity substrate. The bottom metal surface of the microstrip substrate and the top metal surface of the cavity substrate had common circular openings underneath the patch. The top microstrip substrate was kept thin in order to minimize the surface wave and the associated feeding network losses. The bottom cavity substrate was relatively thick for bandwidth enhancement. Since the required fractional matching impedance bandwidth of the proposed antenna was inversely proportional to the square root of the dielectric constant, increment of the bottom cavity substrate thickness was used to improve bandwidth. As SIW cavity backed E-shape patch antenna was introduced in [22], which comprised of two substrates with three metal layers. The SIW cavity was formed by metallized via arrays through two substrates and two metal layers at the top and bottom surfaces of the stack structure. The E-shape radiating patch was on the top metal layer. The feeding structure, which is located on the middle metal layer, 
consisted of a feeding via, a feeding stripline, and tunable loaded stubs. The bottom metal layer is a conductor ground plane. Measured results showed that the proposed antenna element had a wide impedance bandwidth of $10.9 \%$.

The fractional $3 \mathrm{~dB}$ axial ratio (AR) bandwidth of a low profile single fed circularly polarized SIW cavity backed antenna is far less than its fractional matching impedance bandwidth. Three dB AR bandwidth becomes the major factor which limits the operating bandwidth of a single fed circularly polarized SIW cavity backed antenna. In order to increase the operating bandwidth, it is preferable to employ multifeed configurations such as a series feed and a parallel feed as feeding networks. A T-junction power divider with equal magnitude and $90^{\circ}$ phase difference outputs was adopted as the two-point parallel feed to excite a single SIW cavity backed cross slot antenna [23]. Its measured $3 \mathrm{~dB} A R$ bandwidth was about two times that of a single point fed SIW cavity backed cross slot antenna. A dual circularly polarized SIW cavity backed cross slot antenna was proposed in [24], in which the single SIW cavity was stimulated by a branch line coupler. The hybrid coupler generated two signals with equal magnitude and quadrature phase. So, it was not only used to improve the AR bandwidth, but also used to obtain the dual circularly polarized radiation. Right-hand circularly polarized or left-hand circularly polarized radiation can be easily achieved by selecting one port as the feed port while terminating the other port with a matched load. Sequential rotated feeding scheme is another widely used feeding network to improve $3 \mathrm{~dB}$ AR bandwidth of circularly polarized antenna array. Four linearly polarized SIW cavity backed slot antennas fed by a microstrip sequential rotated feeding network were presented in [25] and four circularly polarized SIW cavity backed ring slot antennas fed by a SIW sequential rotated feeding network were presented in [26]. By using these multipoint parallel feeding schemes, great enhancement of $3 \mathrm{~dB}$ AR bandwidths and evidently improvement of circular polarization purity were achieved.

\section{Size Reduction of SIW CBA}

The cavity size of a normal SIW cavity backed antenna is about $0.5 \lambda_{0} * 0.5 \lambda_{0}$, which is not suitable for array application. Miniaturization of a radiating slot, as one of the popular size reduction methods of SIW cavity backed antennas, had been investigated by many researchers. A size-reduced SIW cavity backed slot antenna had been proposed in [27], whose slot consisted of a pair of meandered slots. Its size reduction of $50 \%$ was accomplished by inserting a capacitive element into the SIW cavity, which leads the increment of the stored electric energy and nearly unaffected the stored magnetic energy. A cylindrical metal post, through the thickness of the cavity and aligned parallel to the electric field, was implemented as the capacitive element. The post was isolated from the top metal surface of the SIW cavity by using a ring slot. A miniaturized SIW cavity backed slot antenna with dual polarized radiation was discussed in [28]. Two orthogonal feeding lines, which were directly connected to the antenna slot, were used to excite the SIW cavity and to provide the impedance conversion. The two ports are perpendicularly placed to generate two orthogonal polarizations. The size reduction was achieved by meandering the slot line. Another miniaturized SIW cavity backed meandered slot antenna array was shown in [29]. The meandered slot acted as a left-handed (LH) capacitor and vias constructing SIW acted as $\mathrm{LH}$ inductors. Their cooperation leaded to a negative resonance existence in the SIW cavity. Electromagnetic waves propagate below the cutoff frequency of the fundamental mode, which leads to the reduction of the antenna size. Mutual coupling between two adjacent elements of the proposed miniaturized SIW backed meandered slot antenna array was significantly small.

Another popular size-reducing method of SIW cavity backed antennas is achieved by cavity size reduction. A super compact SIW cavity backed slot antenna was introduced in [30]. The backed cavity was miniaturized by folding the cavity using multilayer substrate and meandering the passage from the bottom of the cavity to the radiating slot. A size-reduced cavity backed antenna was proposed in [31] by using half mode substrate integrated waveguide (HMSIW) technique. The backed cavity of the antenna is triangular in shape, which was realized by metallized vias array through the substrate, the bottom ground plane, and the top triangular patch. This triangular cavity was a half mode cavity in which the field distribution was almost half of that in the original SIW cavity. The resonating frequency of the proposed triangular HMSIW cavity is equal to that of its corresponding square SIW one. Radiating field was generated by dominant $\mathrm{TE}_{110}$ cavity mode through a dielectric aperture created by HMSIW. The proposed antenna was reduced to half of its corresponding SIW cavity backed antenna. A quarter-mode substrate integrated waveguide (QMSIW) cavity backed antenna also had been reported in [32]. The QMSIW cavity was the quadrant sector of a square SIW cavity, which is realized by bisecting the HMSIW cavity into two parts along the fictitious quasimagnetic wall. The QMSIW cavity preserved the field distribution of an SIW cavity and leaky wave was achieved from the dielectric aperture. The size of the proposed antenna was about $25 \%$ of that of counterpart SIW cavity backed antenna.

A wearable SIW cavity backed slot antenna was presented in [33], which was implemented with textile materials. The cavity substrate was closed-cell expanded rubber protective foam typically used in firefighter suits. Its top and bottom metal layers were implemented using a conductive fabric. The proposed textile antenna exhibited high robustness against bending and low influence on the human body, and suited for on-body use. Another wearable textile antenna was proposed in [34] based on an HMSIW cavity. Its top and bottom metal surfaces were formed of silver-coated fabric and HMSIW cavity was implemented through via-walls made of a conductive yarn. The antenna radiated through a thin aperture. The ground plane provided a good isolation for the human body in wearable application.

\section{Gain Improvement of SIW CBA}

In many practical applications, high gain radiation is required. Single gain improved antenna element and antenna array designs are the most common methods to meet this 
requirement. A gain enhanced SIW cavity backed dual slot antenna was presented in [35]. The two parallel slots close to the cavity edges and perpendicular to the GCPW feeding line were used as radiating elements. When $\mathrm{TE}_{120}$ resonance was successfully excited in the SIW cavity, the field drastic transition domains were located at the cavity center and the cavity edges. Effective electromagnetic waves were radiated from the two parallel slots and then a high gain radiation was generated when the radiated waves were superposed in phase in the farfield. A gain enhanced cavity backed slot antenna using high order cavity resonance was proposed in [36]. A GCPW line located at one diagonal line of the SIW cavity was used as the feeding element to excite the $\mathrm{TE}_{220}$ resonance in the cavity. Triple parallel slots, parallel to the two cavity edges, were used as the radiating elements. One of the triple parallel slots was etched at one center line of the SIW cavity. The other two slots were symmetrically distributed about the SIW cavity center line and they were close to the SIW cavity walls. Three groups of tuning posts located at the two center lines of the SIW cavity were used as the auxiliary tuning elements to excite the required $\mathrm{TE}_{220}$ resonance. This design method can be extended to be used at the higher order cavity resonances, such as $\mathrm{TE}_{230}, \mathrm{TE}_{330}$, and $\mathrm{TE}_{440}$, to get a much higher gain radiation. An SIW cavity backed slot array antenna was introduced in [37]. The proposed antenna had two substrate layers (microstrip line substrate and cavity substrate) and three metal surfaces. SIW cavity backed slot array was etched on the top metal surface and the microstrip feeding line was located at the bottom metal surface. Energy coupling to the SIW cavity was achieved by means of a coupling slot etched on the middle metal surface. The middle metal surface also served as the ground plane of the microstrip feeding line. The SIW cavity was designed to excite $\mathrm{TE}_{508}$ resonance to the required frequency. Some tuning posts were introduced to suppress the mode $\mathrm{TE}_{706}$, the closest unwanted mode. The tuning posts should be placed in the points, where the electric field associated with the desired mode is null and that of the closest unwanted mode is as high as possible. The number of array elements was determined by the desired gain, which corresponds to a specified cavity mode.

An SIW cavity backed slot antenna array was reported in [38], which was fed by a T-type microstrip power divider network. Comparing to that of a single antenna element, the antenna array gain had been improved of $5.8 \mathrm{~dB}$ by using a $2 *$ 2 array. An SIW cavity backed wide slot antenna array operating at $60 \mathrm{GHz}$ was studied in [39]. The proposed antenna behaved the dual-resonance operating mechanism. Its backed cavities were not only reflectors for the radiating slots but also radiating elements. Measured results showed that the proposed $2 * 4$ antenna array had a gain of $12 \mathrm{dBi}$ and a cross polarization level lower than $25 \mathrm{~dB}$ over its whole operating bandwidth of about $11.6 \%$.

\section{Active SIW CBA}

An active SIW cavity backed antenna is defined as an antenna with active device integrated in the SIW cavity backed antenna. It includes reconfigurable antenna, oscillator antenna, and so forth. An SIW cavity backed slot antenna with a reconfigurable circular polarization was investigated in [40]. A crossed slot etched on the top metal plane was used as a radiator. Its two arms were orthogonal and identical. Two pairs of shorting posts were located at the extended center lines of the cross slot with the same distance. Two pairs of diodes with opposite biased states were used to switch the shorting posts. When one pair of diodes was switched on and the other one was switched off, a perturbation caused by shorting diodes pair was more than that caused by by opening diodes pair. Then the two near degenerate cavity modes, $\mathrm{TM}_{110}$ resonances, were excited. Radiating waves from each arms of the cross slot can be set to have equal magnitude and $90^{\circ}$ phase difference. Then a circularly polarized radiation was produced. By controlling the biased states of the two pairs of diodes, a reconfigurable circular polarization of the left hand or the right hand can be easily achieved. A novel varactor-tuned SIW cavity backed slot antenna was presented in [41]. It used four varactors mounted on a dumbbell-shaped slot, which was etched on one metal surface of the SIW cavity. The operating frequency of the proposed antenna was tuned from $5.5 \mathrm{GHz}$ to $7.6 \mathrm{GHz}$, preserving the radiation pattern over the entire tuning range. Its frequency reconfigurability was easily achieved without additional bias network. A novel tunable SIW cavity backed slot antenna was proposed in [42]. Two parallel ferrite slabs with the same height of the SIW cavity were inserted into the cavity, which were also parallel to the cavity edges. The magnets were used to supply the bias magnetic field. The field strength is changed by tuning the distance between the two magnets along the cavity height direction. The operating frequency of the proposed antenna was tuned by means of a magnetic biasing field.

A tunable SIW cavity backed active slot antenna oscillator was investigated in [43]. A more compact radiating slot, with the shape of dog bone, was etched at the bottom metal layer of the SIW cavity. One via of the SIW cavity wall was removed and a varactor diode was connected between the SIW cavity top metal layer and a radial stub in order to obtain a tunable capability. Tuning of the varactor bias voltage produces a change in the loading of the cavity and consequently modifies its resonance frequency. Another tunable SIW cavity backed active patch antenna oscillator was studied in [44]. A rectangular ring slot was etched on the bottom metal surface of the SIW cavity to form a patch antenna. A GCPW feeding line on the top metal surface of the SIW cavity was extended inside the cavity, and a slot was etched perpendicular to the GCPW feeding line on the same metal surface. The gate of the active device was connected to the cavity's feeding line. The oscillator was self-biased by placing two resistors from the source terminals to ground. Stubs were used in the feeding line to eliminate the unwanted parasitic oscillations and additionally fine-tune the oscillation frequency.

\section{Conclusions}

A review of low profile cavity backed antennas based on SIW technique has been presented in this paper. Recent advances of SIW cavity backed antennas have been described. Performance improvements of SIW cavity backed antenna have been investigated, including bandwidth enhancement, size 
reduction, and gain improvement. Active antennas comprised of SIW cavity backed antennas also have been studied.

\section{Acknowledgments}

This work was supported in part by the National Basic Research Program of China under Contract 2010CB327403, the National Science and Technology Major Project of China under Contract 2010ZX03007-001-01, the NSFC under Contract 61372020, the ZJNSF under Contract R1110003, and the FANEDD under Contract 201045.

\section{References}

[1] J. Sarrazin, Y. Mahé, S. Avrillon, and S. Toutain, "Investigation on cavity/slot antennas for diversity and MIMO systems: the example of a three-port antenna," IEEE Antennas and Wireless Propagation Letters, vol. 7, pp. 414-417, 2008.

[2] M. Manteghi and Y. Rahmat-Samii, "Multiport characteristics of a wide-band cavity backed annular patch antenna for multipolarization operations," IEEE Transactions on Antennas and Propagation, vol. 53, no. 1, pp. 466-474, 2005.

[3] Q. L. Guo and L. S. Ling, "Circularly polarized antenna based on dual-mode circular SIW cavity," in Proceedings of the International Conference on Microwave and Millimeter Wave Technology (ICMMT '08), vol. 13, pp. 1077-1079, Nanjing, China, April 2008.

[4] G. Q. Luo, Z. F. Hu, L. X. Dong, and L. L. Sun, "Planar slot antenna backed by substrate integrated waveguide cavity," IEEE Antennas and Wireless Propagation Letters, vol. 7, pp. 236-239, 2008.

[5] G. Q. Luo, L. L. Sun, and L. X. Dong, "Single probe fed cavity backed circularly polarized antenna," Microwave and Optical Technology Letters, vol. 50, no. 11, pp. 2996-2998, 2008.

[6] L. Yan, W. Hong, G. Hua, J. Chen, K. Wu, and T. J. Cui, "Simulation and experiment on SIW slot array antennas," IEEE Microwave and Wireless Components Letters, vol. 14, no. 9, pp. 446448, 2004.

[7] J. Lacik and T. Mikulasek, "Substrate integrated waveguide rectangular ring slot antenna," in Proceedings of the 13th International Conference on Electromagnetics in Advanced Applications (ICEAA '11), pp. 1164-1167, September 2011.

[8] S. Pan and F. Capolino, "Design of a CMOS on-chip slot antenna with extremely flat cavity at $140 \mathrm{GHz}$," IEEE Antennas and Wireless Propagation Letters, vol. 10, pp. 827-830, 2011.

[9] G. Q. Luo, Z. F. Hu, Y. Liang, L. Y. Yu, and L. L. Sun, "Development of low profile cavity backed crossed slot antennas for planar integration," IEEE Transactions on Antennas and Propagation, vol. 57, no. 10, pp. 2972-2979, 2009.

[10] D. Kim, J. W. Lee, C. S. Cho, and T. K. Lee, "X-band circular ring-slot antenna embedded in single-layered SIW for circular polarisation," Electronics Letters, vol. 45, no. 13, pp. 668-669, 2009.

[11] J. Lacik, "Circularly polarized SIW square ring-slot antenna for X-band applications," Microwave and Optical Technology Letters, vol. 54, no. 11, pp. 2590-2594, 2012.

[12] M. H. Awida and A. E. Fathy, "Substrate-integrated waveguide Ku-band cavity-backed $2 \times 2$ microstrip patch array antenna," IEEE Antennas and Wireless Propagation Letters, vol. 8, pp. 1054-1056, 2009.
[13] M. H. Awida, E. Elkhouly, and A. E. Fathy, "Low-cost highefficiency substrate-integrated cavity-backed single element antenna," in Proceedings of the IEEE International Symposium on Antennas and Propagation (AP-S/URSI '10), pp. 1-4, Toronto, Canada, July 2010.

[14] D.-Y. Kim, J. W. Lee, T. K. Lee, and C. S. Cho, "Design of SIW cavity-backed circular-polarized antennas using two different feeding transitions," IEEE Transactions on Antennas and Propagation, vol. 59, no. 4, pp. 1398-1403, 2011.

[15] T. Mikulasek and J. Lacik, "Circularly polarized microstrip patch antenna fed by substrate integrated waveguide," in Proceedings of the 6th European Conference on Antennas and Propagation, pp. 2380-2383, March 2012.

[16] G. Q. Luo, Z. F. Hu, W. J. Li, X. H. Zhang, L. L. Sun, and J. F. Zheng, "Bandwidth-enhanced low-profile cavity-backed slot antenna by using hybrid SIW cavity modes," IEEE Transactions on Antennas and Propagation, vol. 60, no. 4, pp. 1698-1704, 2012.

[17] S. Yun, D. Kim, and S. Nam, "Bandwidth enhancement of cavity-backed slot antenna using a via-hole above the slot," IEEE Antennas and Wireless Propagation Letters, vol. 11, pp. 10921095, 2012.

[18] S. Yun, D. Kim, and S. Nam, "Bandwidth and efficiency enhancement of cavity-backed slot antenna using a substrate removal," IEEE Antennas and Wireless Propagation Letters, vol. 11, pp. 1458-1461, 2012.

[19] K. J. Lee, J. A. Lee, and M. Kim, "Multilayer dielectric cavity antenna design for wide bandwidth," Microwave and Optical Technology Letters, vol. 54, no. 9, pp. 2046-2049, 2012.

[20] G. Q. Luo, "Low profile cavity backed antennas based on substrate integrated waveguide technology," in Proceedings of the IEEE Asia-Pacific Conference on Antennas and Propagation, pp. 275-276, Singapore, August 2012.

[21] M. H. Awida, S. H. Suleiman, and A. E. Fathy, "Substrateintegrated cavity-backed patch arrays: a low-cost approach for bandwidth enhancement," IEEE Transactions on Antennas and Propagation, vol. 59, no. 4, pp. 1155-1163, 2011.

[22] W. Yang and J. Zhou, "Wideband low profile substrate integrated waveguide cavity backed E-shaped patch antenna," IEEE Antennas and Wireless Propagation Letters, vol. 12, pp. 143-146, 2013.

[23] G. Q. Luo, Z. F. Hu, X. H. Zhang, L. X. Dong, and L. L. Sun, "A Tjunction power divider FED circularly polarized cavity backed slot antenna," Microwave and Optical Technology Letters, vol. 54, no. 1, pp. 107-109, 2012.

[24] G. Q. Luo, P. Li, Z. C. Zheng, and L. L. Sun, "A dual circularly polarized antenna with improved axial ratio bandwidth," in Proceedings of the China-Japan Joint Microwave Conference (CJMW'11), pp. 179-182, Hangzhou, China, April 2011.

[25] G. Huang and G. Q. Luo, "Circularly polarized cavity backed slot antenna with sequential-rotated feeding," Chinese Journal of Electron Devices, vol. 34, no. 2, pp. 146-149, 2011.

[26] E. Jung, J. W. Lee, T. K. Lee, and W. K. Lee, "SIW-based array antennas with sequential feeding for X-band satellite communication," IEEE Transactions on Antennas and Propagation, vol. 60, no. 8, pp. 3632-3639, 2012.

[27] C. A. T. Martinez, J. C. B. Reyes, O. A. N. Manosalva, and N. M. P. Traslavina, "Volume reduction of planar substrate integrated waveguide cavity-backed antennas," in Proceedings of the 6th European Conference on Antennas and Propagation, pp. 29192923, March 2012.

[28] Y. Dong and T. Itoh, "Miniaturized cavity-backed dualpolarized slot antenna," in Proceedings of the IEEE Antennas and Propagation Society International Symposium, pp. 1-2, July 2012. 
[29] P. Loghmannia, M. Kamyab, M. Ranjbar Nikkhah, and R. Rezaiesarlak, "Miniaturized low-cost phased-array antenna using SIW slot elements," IEEE Antennas and Wireless Propagation Letters, vol. 11, pp. 1434-1437, 2012.

[30] W. Hong and K. Sarabandi, "Platform embedded slot antenna backed by shielded parallel plate resonator," IEEE Transactions on Antennas and Propagation, vol. 58, no. 9, pp. 2850-2857, 2010.

[31] S. A. Razavi and M. H. Neshati, "Development of a low profile circularly polarized cavity backed antenna using HMSIW technique," IEEE Transactions on Antennas and Propagation, vol. 61, no. 3, pp. 1041-1047, 2013.

[32] C. Jin, R. Li, A. Alphones, and X. Bao, "Quarter-mode substrate integrated waveguide and its application to antennas design," IEEE Transactions on Antennas and Propagation, vol. 61, no. 6, pp. 2921-2928, 2013.

[33] R. Moro, S. Agneessens, H. Rogier, and M. Bozzi, "Wearable textile antenna in substrate integrated waveguide technology," Electronics Letters, vol. 48, no. 16, pp. 985-987, 2012.

[34] T. Kaufmann and C. Fumeaux, "Wearable textile half-mode substrate integrated cavity antenna using embroidered vias," IEEE Antennas and Wireless Propagation Letters, vol. 12, pp. 805-808, 2013.

[35] J. X. Li, G. Q. Luo, Y. Liang, W. J. Li, L. X. Dong, and L. L. Sun, "Cavity backed dual slot antenna for gain improvement," Microwave and Optical Technology Letters, vol. 52, no. 12, pp. 27672769, 2010.

[36] G. Q. Luo, X. H. Zhang, L. X. Dong, W. J. Li, and L. L. Sun, "A gain enhanced cavity backed slot antenna using high order cavity resonance," Journal of Electromagnetic Waves and Applications, vol. 25, no. 8-9, pp. 1273-1279, 2011.

[37] M. J. Jiménez-Fernández, R. Torres-Sánchez, and P. Otero, "Cavity-backed slot array antenna in substrate-integrated waveguide technology," Microwave and Optical Technology Letters, vol. 53, no. 9, pp. 2105-2108, 2011.

[38] K. Jiang, G. Q. Luo, W. J. Li, G. Huang, P. Li, and L. L. Sun, "A $2 * 2$ antenna array consisting of low profile cavity backed slot antennas," in Proceedings of the China-Japan Joint Microwave Conference (CJMW'11), pp. 74-76, Hangzhou, China, April 2011.

[39] K. Gong, Z. N. Chen, X. Qing, P. Chen, and W. Hong, "Substrate integrated waveguide cavity-backed wide slot antenna for $60 \mathrm{GHz}$ bands," IEEE Transactions on Antennas and Propagation, vol. 60, no. 12, pp. 6023-6026, 2012.

[40] G. Q. Luo and L. L. Sun, "A reconfigurable cavity backed antenna for circular polarization diversity," Microwave and Optical Technology Letters, vol. 51, no. 6, pp. 1491-1493, 2009.

[41] A. H. Mohammadi and K. Fororaghi, "A varactor-tuned substrate-integrated cavity-backed dumbbell slot antenna," in Proceedings of the 15th International Symposium on Antenna Technology and Applied Electromagnetics, pp. 1-3, June 2012.

[42] L. Tan, R. Wu, C. Wang, and Y. Poo, "Magnetically tunable ferrite loaded SIW antenna," IEEE Antennas and Wireless Propagation Letters, vol. 12, pp. 273-275, 2013.

[43] F. Giuppi, A. Georgiadis, A. Collado, M. Bozzi, and L. Perregrini, "Tunable SIW cavity backed active antenna oscillator," Electronics Letters, vol. 46, no. 15, pp. 1053-1055, 2010.

[44] F. Giuppi, A. Georgiadis, A. Collado, M. Bozzi, S. Via, and L. Perregrini, "An X band, compact active cavity backed patch oscillator antenna using a substrate integrated waveguide (SIW) resonator," in Proceedings of the IEEE International Symposium on Antennas and Propagation, pp. 1-4, Toronto, Canada, July 2010. 

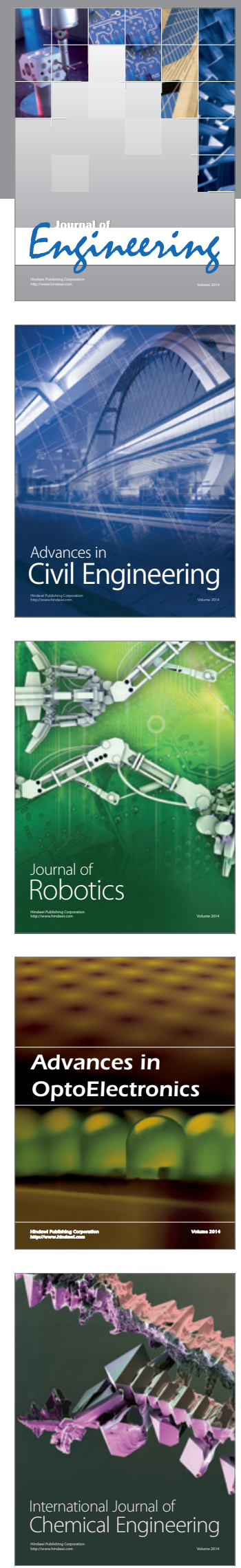

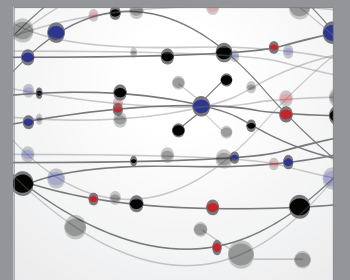

The Scientific World Journal
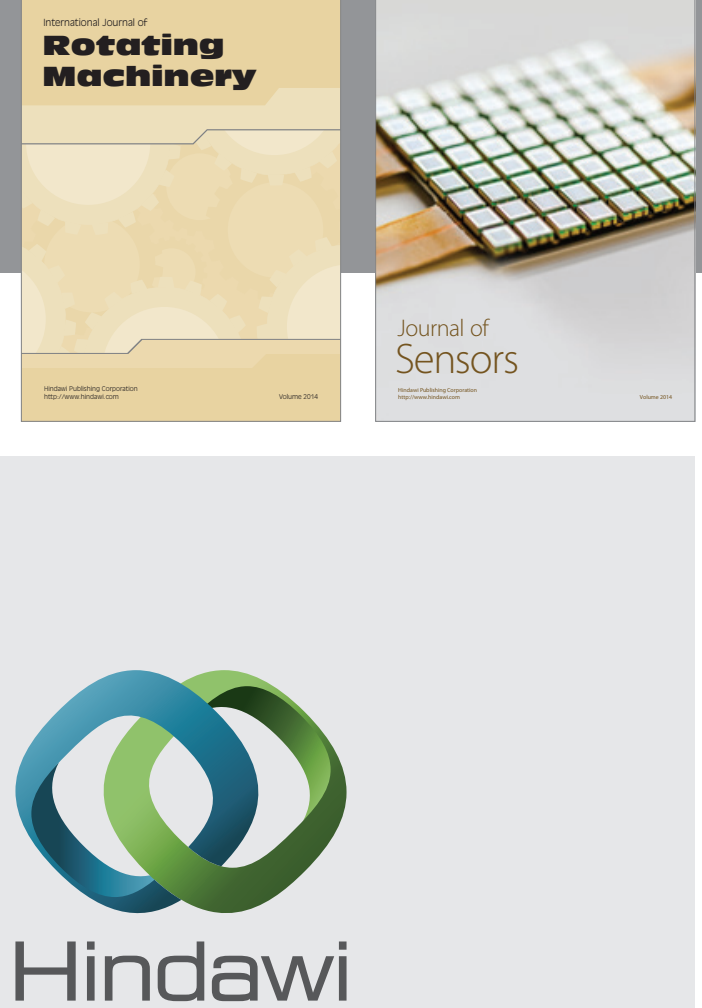

Submit your manuscripts at http://www.hindawi.com
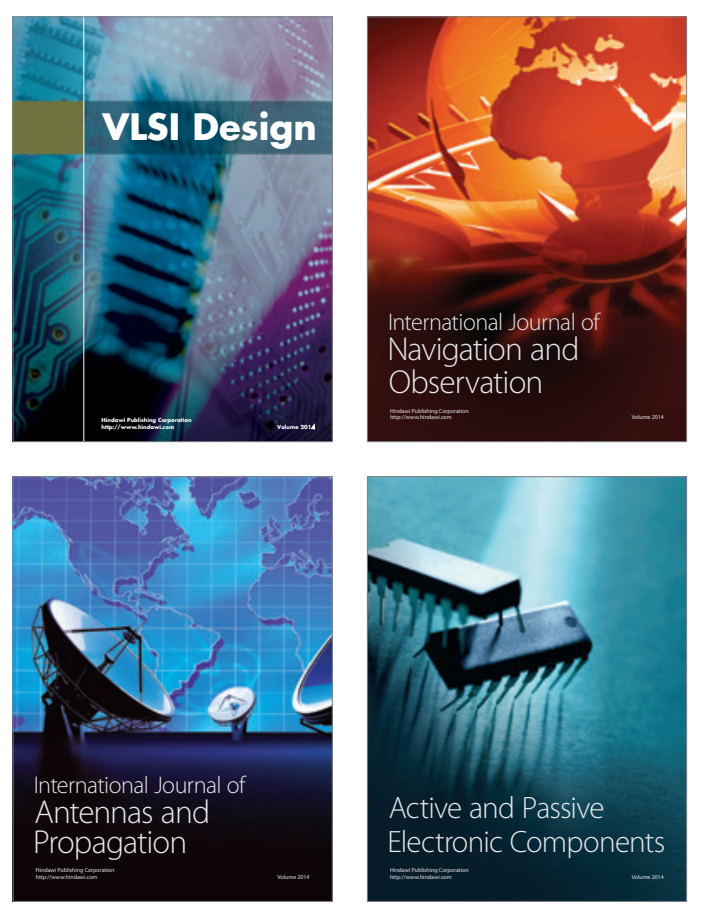
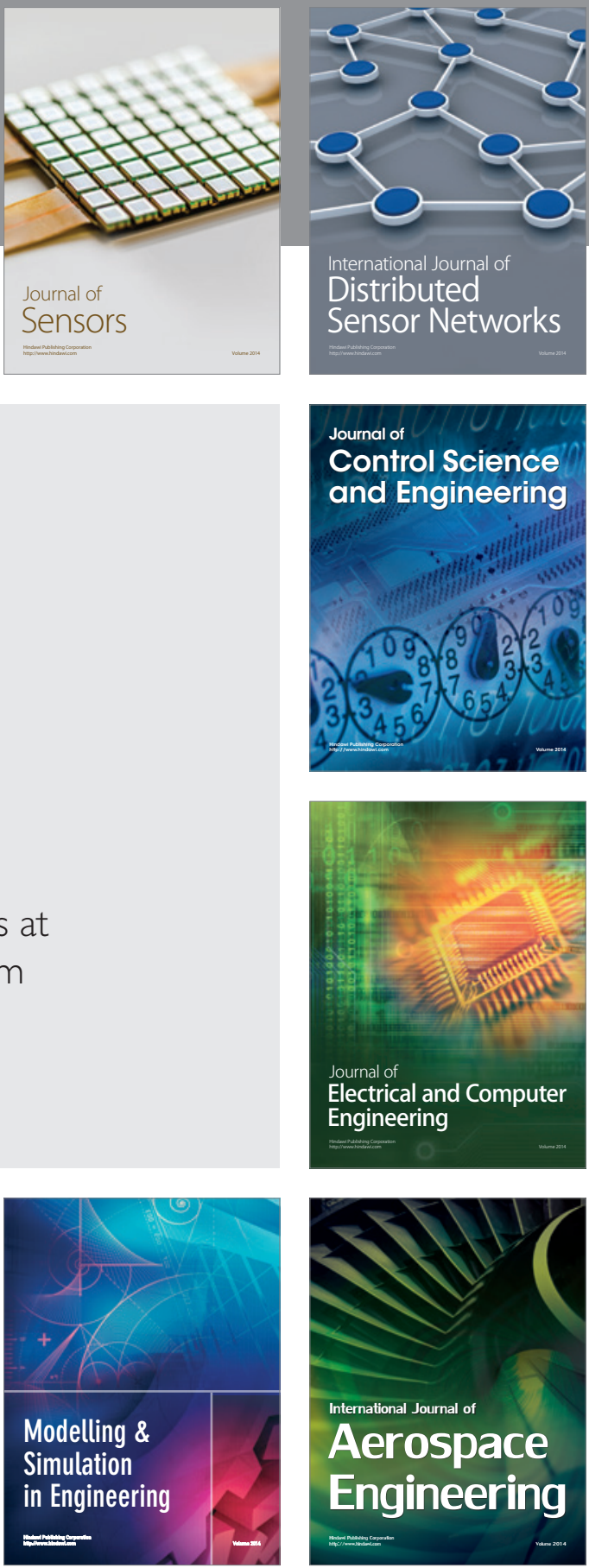

Journal of

Control Science

and Engineering
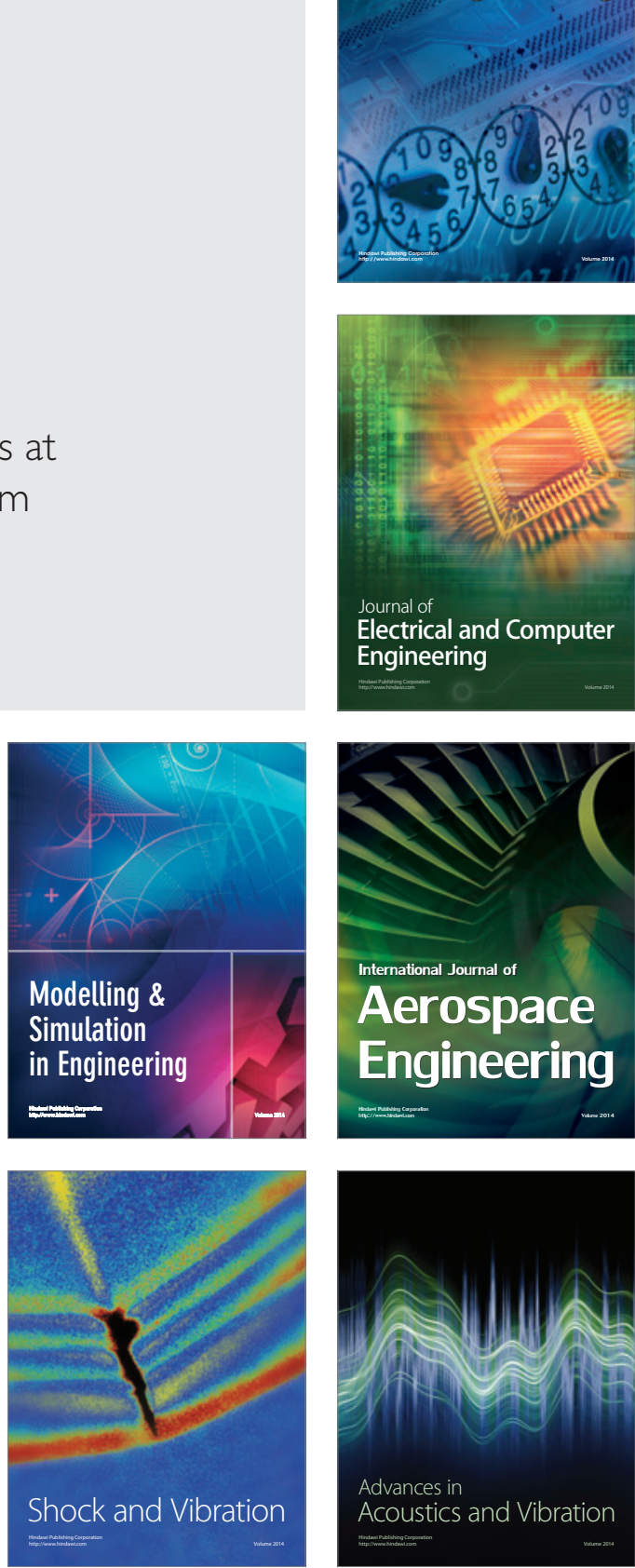